\title{
Percepções sobre obesidade em escolares decorrentes de ação educativa interdisciplinar
}

\author{
Perceptions about obesity in schoolchildren due to \\ interdisciplinary educational action
}

\author{
Renato da Costa Teixeira', Elen Lemões Mota de Almeida² \\ 'Universidade do Estado do Pará, Belém (PA), Brasil. \\ ${ }^{2}$ Faculdade Adventista da Amazônia, Benevides (PA), Brasil.
}

Recebido: Ago. 09, 2017 Aceito: Abr. 03, 2019

\section{COMO CITAR ESTE ARTIGO Teixeira RC, Almeida ELM. Percepções sobre obesidade em escolares decorrentes de ação educativa interdisciplinar. Interdisciplinary Journal of Health Education. 2018 Jan-Dez;3(1-2):16-23. https://doi. org/10.4322/ijhe.2018.006. \\ CORRESPONDÊNCIA \\ Renato da Costa Teixeira \\ Departamento de Ciências do \\ Movimento Humano, Universidade do Estado do Pará \\ TV Perebebuí, 2623, Marco, CEP $66087-$ 662 , Belém (PA), Brasil \\ teixeirarenato@globo.com}

\section{FONTE DE FINANCIAMENTO}

Nenhuma.

\section{CONFLITO DE INTERESSE}

Os autores declararam não

haver conflitos de interesse.

O estudo foi realizado no Colégio da Faculdade Adventista da Amazônia, Benevides, Pará (PA), Brasil.

Todos os autores leram e aprovam a versão final submetida ao Interdisciplinary Journal of Health Education (IJHE).

\section{RESUMO}

Contextualização: A escola é um espaço promotor de saúde. A interdisciplinaridade na escola é uma estratégia onde pode ocorrer a promoção de saúde. Temas transversais, como a obesidade, pode ser conduzido através das áreas de direitos das crianças e adolescentes, educação alimentar e nutricional, saúde, vida familiar e social, educação para o consumo, fomentando ações interdisciplinares de modo contextualizado, buscando não fragmentar o conhecimento em blocos rígidos, para que a educação se constitua em um meio de transformação social. Para tanto, o professor deve levar seus alunos a refletir sobre temáticas atuais. A alimentação saudável favorece a criação de bons hábitos alimentares para toda a vida. O presente tem como objetivo relatar uma atividade educativa interdisciplinar com escolares da primeira série do Ensino Médio de uma escola particular em que o tema central foi a obesidade, tendo sido aprovado pelo Comitê de Ética em Pesquisa da Universidade do Estado do Pará antes de seu início. Descrição da experiência: Foi utilizado um questionário em que se buscou dados gerais dos participantes e o que entendiam sobre obesidade, sendo os resultados apresentados em quadros. Fizeram parte da ação 90 alunos. $O$ termo mais evocado pelos participantes antes da ação educativa foi "gordura" com 50 evocações. Após esta ação foi "Acúmulo de gordura" com 41 evocações seguido de prevenção com 40 evocações. O termo "prevenção" que não havia sido evocado pelos participantes antes da ação educativa assim como a palavra "morte" passaram a fazer parte das evocações após a ação educativa. Considerações finais: O projeto interdisciplinar se mostrou um colaborador educativo no processo de educação em saúde.

palavras-chave: Adolescente. Obesidade. Instituições de ensino. Aprendizagem por associação.

\section{ABSTRACT}

Contextualization: A school is a place for health promotion, and interdisciplinarity at school is a strategy to realize it. Cross-cutting themes, such as obesity, might be examined in areas such as children's rights, food and nutrition education, health, family and social life, and consumer education, fostering interdisciplinary actions in a contextualized way, which prevents knowledge from being fragmented into blocks, so that education becomes a means of social transformation. Teacher should lead their students in reflecting on current themes. A healthy diet favors the creation of good eating habits throughout one's life. This study aims to report an interdisciplinary educational activity with high school students of a private school in which the central theme was obesity, which was approved by the Research Ethics Committee of the University of the State of Pará before it was implemented. Description of the experience: A questionnaire was used to obtain general data about the participants and what they understood about obesity, and the results are presented in tables. Ninety students took part in the action. The most frequently mentioned term by participants before the educational action was "fat," with 50 mentions. After this action was "accumulation of fat" came second, with 41 mentions, followed by "prevention," with 40 mentions. The terms "prevention" and "death," which had not been mentioned by the participants before the educational action, were mentioned following its implementation. Final considerations: The interdisciplinary project was an educational collaborator in the health education process. KEYWORDS: Adolescent. Obesity. Educational institutions. Learning by Association. 


\section{Introdução}

Uma alimentação saudável na adolescência, favorece a criação e manutenção de bons hábitos alimentares para toda a vida, concedendo peso e desenvolvimento adequados de massa óssea e muscular, nesse período tão necessário, devido ao aumento de atividades estudantis e/ou profissionais ${ }^{1}$.

Mudanças biológicas, sociais e de comportamento que ocorrem nessa etapa de vida afetam de forma significativa os hábitos alimentares, havendo troca de alimentos saudáveis por outros, podendo repercutir na saúde do adolescente em curto ou longo prazo ${ }^{2}$.

Adolescência é definida não só como uma fase de transição entre a infância e a idade adulta, mas como um período em que ocorre várias mudanças, uma fase em que são identificados amplos problemas de saúde, nutrição, crescimento físico e acadêmico, podendo ser um período vulnerável à obesidade, devido à falta de atividade física, juntamente com o consumo de uma dieta rica em açúcar, gordura saturada, sal e conteúdo calórico ${ }^{3}$.

Ao ser avaliado hábitos alimentares, preferências alimentares e o padrão alimentar de adolescentes urbanos na India, foram relados padrões alimentares pouco saudáveis e as preferências por fast food, junk food e doces e quando perguntados o que tendem a não comer, responderam vegetais frescos ou cozidos, folhas verdes e frutas ${ }^{3}$.

A Pesquisa de Orçamento Familiar tem mostrado, segundo o Instituto Brasileiro de Geografia e Estatística, que quase metade (49\%) da população brasileira está com excesso de peso, $14,8 \%$ estão obesas e somente 2,7\% apresentam déficit de peso. Esses dados atingem, em grande parte os adolescentes ${ }^{4}$.

Cabe destacar que a prevalência de excesso de peso em adolescentes e crianças brasileiras de seis a dezoito anos de idade triplicou nos últimos anos. Dados da Pesquisa Nacional sobre Demografia e Saúde ${ }^{5}$ mostraram que $21,6 \%$ dos adolescentes entre 15 e 19 anos estavam com excesso de peso, sendo que $4 \%$ deles se encontravam obesos e apenas $2,2 \%$ estavam com déficit de peso ${ }^{1}$.

As escolas são um dos lugares para intervenção no ganho excessivo de peso ${ }^{6}$ e nos últimos anos, a escola passou a ser vista como um ambiente favorável à promoção de saúde e foi estimulada a caminhar na busca de um bem-estar individual e coletivo para os alunos, professores e servidores.

A "Escola Promotora de Saúde" se tornou uma importante estratégia adotada por vários países, estados e municípios ${ }^{6}$, tendo o objetivo de alcançar estilos de vida saudáveis para toda a população por meio do desenvolvimento de ambientes que conduzam à promoção de saúde, formando futuras gerações com conhecimentos, habilidades e atitudes necessárias para promover e cuidar de sua saúde, de sua família e da comunidade ${ }^{7}$.

Tais estratégias estão incluídas em metodologias interdisciplinares e são práticas desafiadoras na organização curricular, na medida em que exigem uma articulação e um diálogo entre os conhecimentos.

A Base Nacional Comum Curricular (BNCC) recentemente lançada, é um documento de caráter normativo que define o conjunto de aprendizagens essenciais que todos os alunos devem desenvolver ao longo das etapas e modalidades da Educação Básica ${ }^{8}$.

Esta estabelece que cabe as escolas incorporarem a seus currículos a abordagem de temas contemporâneos que afetam a vida humana em escala local, regional e global, preferencialmente de forma transversal e integradora, dentre eles: direitos das crianças e adolescentes, educação alimentar e nutricional, saúde, vida familiar e social, educação para o consumo.

Com base no exposto, o presente estudo buscou investigar as percepções de alunos da $1^{a}$ série do ensino médio de uma escola privada sobre obesidade pré e pós uma ação educativa de caráter interdisciplinar. 


\section{Descrição da experiência}

Trata-se do relato de uma atividade desenvolvida com alunos da primeira série do ensino médio com aplicação de questionários em que se buscou dados gerais dos participantes e o que entendiam sobre obesidade, seguidos de atividades interdisciplinares. Obteve-se a aprovação do Comitê de Ética em Pesquisa, do Centro de Ciências Biológicas e da Saúde - UEPA sob o parecer 1.547.064.

A atividade foi realizada no Colégio da Faculdade Adventista da Amazônia, um colégio particular localizado no município de Benevides, estado do Pará durante todo o primeiro semestre letivo de 2016. A escola possui 270 alunos matriculados no ensino médio e o presente estudo foi desenvolvido de forma experimental com os alunos da $1^{\text {a }}$ série do Ensino Médio (que tem um total de 90 alunos matriculados) para depois ser realizado nas demais séries.

No que se refere aos procedimentos para o desenvolvimento da atividade, foram desenvolvidos em 6 etapas descritas a seguir:

Etapa 1: Reunião com os professores do colégio, para explanar o estudo, os procedimentos de coleta de dados e o desenvolvimento das ações educativas.

Etapa 2: Apresentação do estudo aos alunos, com entrega do Termo de Consentimento Livre e Esclarecido (TCLE), para ser assinado pelos responsáveis e do Termo de Assentimento da Pesquisa, concordando em participar da mesma.

Etapa 3: Após a assinatura dos termos houve o preenchimento pelos alunos de um questionário na Plataforma SURVIO no laboratório de informática, em que se buscava dados gerais (sexo e idade), dados sobre a atividade física e sobre a percepção dos alunos sobre a obesidade. Neste havia o comando: "Escreva até 5 palavras que lhe vêm à mente ao ler ou ouvir a expressão OBESIDADE".

Etapa 4: Realização de encontros pedagógicos com os professores das disciplinas de Artes, Biologia, Educação Física, Filosofia, Inglês, Matemática, Redação e Química, em que foi apresentado a importância da interdisciplinaridade e planejado ações com participação de uma endocrinologista que conversou sobre obesidade e como abordar este tema com os adolescentes de uma maneira que não fosse cobrança.

Professores juntamente com a nutricionista do Colégio analisaram as respostas ao item do questionário preenchido pelos alunos em que eles respondiam o que gostariam de saber sobre obesidade, para a partir de então traçar as metodologias que iriam utilizar e em que momentos das suas aulas iriam realizar as intervenções Pedagógicas.

Nesta questão, foram levantados pelos alunos os seguintes questionamentos: (i) porque as pessoas não conseguem controlar a ansiedade/compulsão pela alimentação, principalmente adolescentes; (ii) medidas de prevenção; (iii) efeitos que a doença pode causar no organismo; (iv) tratamentos atuais; (v) taxas de obesidade dos adolescentes no Brasil e no mundo; (vi) a obesidade é transferida por hereditariedade; (vii) efeitos psicológicos que a obesidade pode causar nos adolescentes; (viii) como os obesos se sentem; (ix) origem da palavra obesidade e em que momento da história começou a debater o assunto.

Etapa 5: Encontros interdisciplinares programados de acordo com o que os alunos gostariam de saber sobre obesidade, seguindo a sequência exposta no quadro 1 a seguir:

Nesses encontros interdisciplinares, fazia-se com que os alunos além de refletirem suas práticas alimentares, pudessem em conjunto com seus colegas discutirem suas práticas quanto em relação ao outro.

Etapa 6: Ao fim do semestre letivo de 2016 os alunos preencheram novamente o questionário na Plataforma Survio durante as aulas na disciplina de Informática.

Para análise das palavras evocadas, foi verificado a frequência absoluta e relativa de cada uma delas antes e após a ação educativa. 
Quadro 1. Sequência de encontros interdisciplinares realizados com os alunos da primeira série do ensino medio da Faculdade Adventista da Amazônia.

\begin{tabular}{|c|c|c|c|}
\hline Tema & Estratégia & Conteúdo & Disciplina \\
\hline $\begin{array}{l}\text { Escolhas alimentares, } \\
\text { Fast food e consumo } \\
\text { inconsequente }\end{array}$ & $\begin{array}{l}\text { Vídeos e reportagens atuais } \\
\text { (Geração Coca-cola) } \\
\text { Debate com os alunos } \\
\text { Redação sobre o tema }\end{array}$ & $\begin{array}{l}\text { Narração e Produção } \\
\text { textual }\end{array}$ & Redação \\
\hline Bullying & $\begin{array}{l}\text { Discussão sobre o tema } \\
\text { Peça teatral }\end{array}$ & Ética e moral & Filosofia \\
\hline $\begin{array}{l}\text { O efeito da ingestão de } \\
\text { gorduras no organismo } \\
\text { e doenças cardíacas e } \\
\text { fatores de risco }\end{array}$ & & Lipídios & Biologia \\
\hline $\begin{array}{l}\text { Reportagem: como o } \\
\text { açúcar é processado? }\end{array}$ & $\begin{array}{l}\text { Discussão em sala e estudo de } \\
\text { caso }\end{array}$ & Ligações químicas & Quimica \\
\hline $\begin{array}{l}\text { Obesidade, sobrepeso e } \\
\text { peso ideal }\end{array}$ & $\begin{array}{l}\text { Com os dados do IMC dos } \\
\text { alunos de } 2016 \text { fornecidos } \\
\text { pelos professores de Educação } \\
\text { Física, foi elaborado gráficos } \\
\text { e comparado com tabelas } \\
\text { internacionais a porcentagem de } \\
\text { alunos do colégio da FAAMA } \\
\text { com Obesidade, sobrepeso e peso } \\
\text { ideal. } \\
\text { Divulgação dados através de } \\
\text { banners }\end{array}$ & $\begin{array}{l}\text { Qualidade de vida, } \\
\text { sedentarismo, } \\
\text { pratica regular de } \\
\text { exercício } \\
\text { Matemática } \\
\text { financeira, razão } \\
\text { e proporção e } \\
\text { percentagem }\end{array}$ & $\begin{array}{l}\text { Educação Física } \\
\text { Matemática }\end{array}$ \\
\hline $\begin{array}{l}\text { Hábitos alimentares no } \\
\text { Estados Unidos }\end{array}$ & $\begin{array}{l}\text { Interpretação de texto } \\
\text { Videodocumentario: Super Size } \\
M e: \text { a dieta do palhaço }\end{array}$ & $\begin{array}{l}\text { Interpretação de } \\
\text { texto }\end{array}$ & Ingles \\
\hline $\begin{array}{l}\text { Hábitos alimentares } \\
\text { saudáveis e } \\
\text { balanceamento de } \\
\text { nutrientes em cada } \\
\text { refeição }\end{array}$ & $\begin{array}{l}\text { Palestra com a Nutricionista } \\
\text { Quizz (torta na cara) } \\
\text { Degustação de alimentos } \\
\text { saudáveis }\end{array}$ & $\begin{array}{l}\text { Bioquimica e estilo } \\
\text { de vida saudável }\end{array}$ & $\begin{array}{l}\text { Biologia e } \\
\text { Nutrição }\end{array}$ \\
\hline $\begin{array}{l}\text { Produção de materiais } \\
\text { gráficos/publicitário } \\
\text { com objetivo de } \\
\text { divulgação comercial, } \\
\text { institucional, político, } \\
\text { educacional ou artístico }\end{array}$ & $\begin{array}{l}\text { Discussão sobre os elementos } \\
\text { principais que devem estar } \\
\text { presentes na lâmina de } \\
\text { divulgação do Projeto através de } \\
\text { trabalho em grupo de } 5 \text { alunos } \\
\text { (confecção da lamina para as } \\
\text { bandejas da cantina da escola) }\end{array}$ & $\begin{array}{l}\text { Marketing direto e } \\
\text { Midia interna }\end{array}$ & Artes \\
\hline
\end{tabular}

\section{Resultados e impacto}

Participaram da ação 90 alunos, dos quais 54 (60\%) eram do sexo feminino, e 36 (40\%) masculino. Quanto à idade, 4 (4,44\%) possuíam 13 anos; 32 (35,56\%) possuíam 14 anos; 38 (42,22\%) possuíam 15 anos; 14 (15,56\%) possuíam 16 anos; e 2 (2,22\%) possuíam 17 anos.

Das palavras evocadas pelos aluno, $65 \%$ relacionaram a obesidade com problemas de saúde (física e psicológica) enquanto cerca de $17 \%$ relacionaram obesidade com alimentação e os demais citaram palavras preconceituosas.

Em um estudo sobre a percepção sobre obesidade em adolescentes de escolas públicas do Distrito Federal, verificou-se que em relação às causas da obesidade, a maioria dos adolescentes concordaram que obesidade representa ter estilo de vida pouco saudável e está relacionado com a ingestão de frituras ou comidas gordurosas e comer em excesso?.

Os autores analisaram também dados sobre a percepção dos adolescentes em relação às consequências da obesidade. A maioria afirmou que obesos sofrem discriminação/bullying, entretanto discordaram que a obesidade cause doenças ou morte 9 . 
A veiculação de imagens de pessoas obesas em materiais educativos atraem a atenção para este grave problema de saúde, no entanto, a exposição dessas imagens pode estimular o preconceito contra pessoas com obesidade ${ }^{10}$.

Adolescentes não relacionam a prevenção da saúde com a alimentação, uma vez que se sentem invencíveis e tem uma visão muito otimista e esperançosa sobre si próprios ${ }^{11}$.

Um estudo com adolescentes de três escolas públicas de Fortaleza, apontou que para os entrevistados obesidade é apenas estar levemente acima do peso $^{12}$. Neste mesmo estudo ao buscar a percepção dos alunos quanto à obesidade na adolescência, foi evidenciado que $5 \%$ consideraram ser normal. Os autores chamam a atenção que escola e pais devem trabalhar em conjunto para mudar esta concepção (obesidade ser normal).

Antes da ação educacional, foram evocadas um total de 52 palavras diferentes descritas e após a ação educacional, os alunos evocaram 23 palavras diferentes, descritas e comparadas com as evocadas antes da ação educacional no quadro 2 :

Obesidade para os alunos participantes do prenete estudo, antes da ação educativa tinha muitos significados (alguns dos quais jocosos como por exemplo: preguiça; fofinho; barrigão; espaçoso; roupa GGG e bola). Após a ação educativa passou a ter menos

Quadro 2. Palavras evocadas, frequência absoluta e relativa das evocações de alunos da primeira série do ensino médio da Faculdade Adventista da Amazônia antes e após ação educativa.

\begin{tabular}{|c|c|c|c|c|c|c|}
\hline & \multicolumn{3}{|c|}{ Pré ação educativa } & \multicolumn{3}{|c|}{ Pós ação educativa } \\
\hline & Palavra & $\begin{array}{l}\text { Frequencia } \\
\text { absoluta das } \\
\text { evocações }\end{array}$ & $\begin{array}{c}\text { Frequencia } \\
\text { relativa das } \\
\text { evocações } \\
(\%)\end{array}$ & Palavra & $\begin{array}{l}\text { Frequencia } \\
\text { absoluta } \\
\text { das } \\
\text { evocações }\end{array}$ & $\begin{array}{c}\text { Frequencia } \\
\text { relativa } \\
\text { das } \\
\text { evocações } \\
(\%)\end{array}$ \\
\hline \multirow{18}{*}{$\begin{array}{l}\text { Núcleo } \\
\text { comum }\end{array}$} & Comida & 25 & 8,99 & Comida & 05 & 1,49 \\
\hline & Excesso de peso & 22 & 7,91 & Excesso de Peso & 16 & 4,78 \\
\hline & Doença & 18 & 6,47 & Doença & 38 & 11,34 \\
\hline & Sedentarismo & 16 & 5,76 & Sedentarismo & 30 & 8,96 \\
\hline & Diabetes & 09 & 3,24 & $\begin{array}{l}\text { Excesso de } \\
\text { açúcar }\end{array}$ & 02 & 0,60 \\
\hline & Bullyng & 09 & 3,24 & Bullyng & 13 & 3,88 \\
\hline & Depressão & 08 & 2,88 & Depressão & 06 & 1,79 \\
\hline & $\begin{array}{l}\text { Falta de } \\
\text { exercício }\end{array}$ & 08 & 2,88 & $\begin{array}{l}\text { Falta de } \\
\text { exercício físico }\end{array}$ & 08 & 2,39 \\
\hline & $\begin{array}{l}\text { Excesso de } \\
\text { gordura }\end{array}$ & 07 & 2,52 & $\begin{array}{l}\text { Acúmulo de } \\
\text { gordura }\end{array}$ & 41 & 12,24 \\
\hline & Fast food & 06 & 2,16 & Fast food & 07 & 2,09 \\
\hline & Pressão alta & 05 & 1,80 & Hipertensão & 03 & 0,90 \\
\hline & $\begin{array}{l}\text { Baixa } \\
\text { autoestima }\end{array}$ & 03 & 1,08 & $\begin{array}{l}\text { Autoestima } \\
\text { baixa }\end{array}$ & 06 & 1,79 \\
\hline & Colesterol & 02 & 0,72 & Colesterol alto & 15 & 4,48 \\
\hline & Preconceito & 02 & 0,72 & Preconceito & 02 & 0,60 \\
\hline & $\begin{array}{l}\text { Problemas } \\
\text { cardíacos }\end{array}$ & 02 & 0,72 & $\begin{array}{l}\text { Doenças } \\
\text { Cardíacas e } \\
\text { respiratórias }\end{array}$ & 20 & 5,97 \\
\hline & Falta de controle & 02 & 0,72 & $\begin{array}{l}\text { Descontrole } \\
\text { alimentar }\end{array}$ & 01 & 0,30 \\
\hline & $\begin{array}{l}\text { Hábitos } \\
\text { alimentares }\end{array}$ & 02 & 0,72 & $\begin{array}{l}\text { Maus hábitos } \\
\text { alimentares }\end{array}$ & 27 & 8,06 \\
\hline & Hereditariedade & 02 & 0,72 & Hereditariedade & 39 & 11,64 \\
\hline
\end{tabular}

Fonte: Pesquisa de campo, 2016. 
Quadro 2. Continuação..

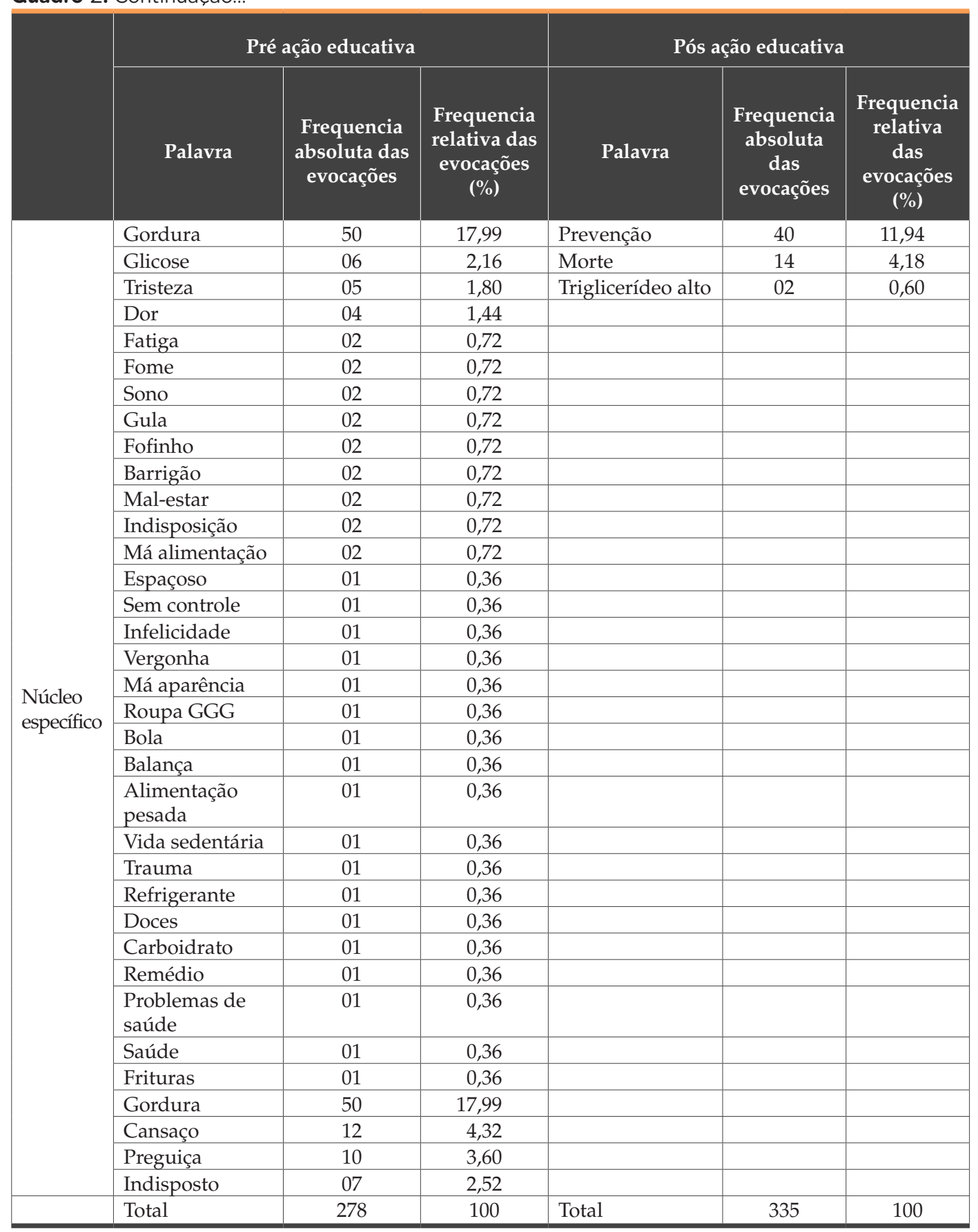

Fonte: Pesquisa de campo, 2016.

significados dos quais a maior parte deles relativos a problemas de saúde. Enquanto antes da ação o significado das palavras relacionadas a saúde foram 208 de um total de 278 evocações $(74,83 \%$ ) após a ação educativa passaram a ser 281 das 335 evocações $(83,88 \%)$. O aumento no número de evocações deve-se ao fato de que no comando os alunos eram orientados a escrever até cinco palavras. Acreditamos que após a ação educativa, por terem mais informações sobre a obesidade, gerou um número maior de evocações.

Na evocação após a ação foi apresentado o termo prevenção que ainda não tinha sido lembrado pelos participantes da pesquisa sendo registrado uma frequência de 40 evocações assim como morte com uma frequência de 14 evocações.

Para os adolescentes a causa de obesidade refletem o senso comum, no que diz respeito ao aumento de tecido adiposo, excesso de gordura corporal causando prejuízo à saúde dos indivíduos ${ }^{13}$. 


\section{ijhe}

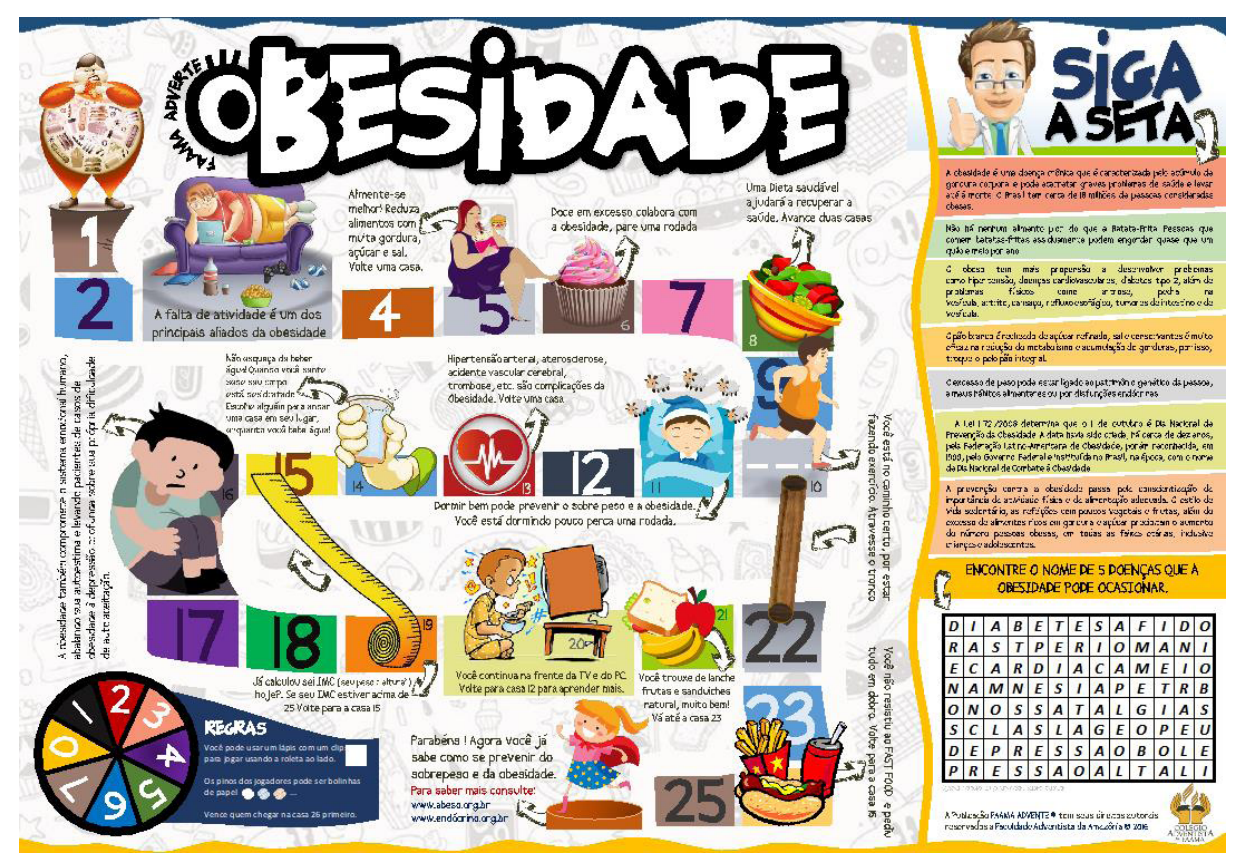

Figura 1. Lâmina de bandeja gerada a partir da ação educativa interdisciplinar por sugestão dos participantes.

No presente estudo foi possível comprovar isso após a ação educativa, quando então relacionaram obesidade a doença, prevenção e morte. No questionário preenchido antes da ação educativa a principal palavra evocada foi gordura enquanto após a ação foi acúmulo de gordura demonstrando que os adolescentes haviam conseguido se aproximar desse senso comum.

\section{Considerações finais}

As ações interdisciplinares estimularam os alunos a discutir sobre suas práticas alimentares e as consequências que estas causam no organismo. Antes da ação educativa, obesidade tinha muitos significados muitos dos quais jocosos. Após a ação passou a representar problema de saúde.

Pode-se afirmar que a discussão sobre promoção de estilo de vida saudável na escola é atual e urgente e que ações educativas podem ser implementadas nos espaços escolares, visando à formação de conceitos e às mudanças de comportamentos.

Sobre o estímulo dos professores em proporcionar discussões entre os alunos, sobre suas próprias práticas alimentares e as consequências no organismo, é possível afirmar, tanto pela forma como ocorreram as intervenções dos alunos nas aulas, como pelo interesse demonstrado durante as atividades interdisciplinares e pelos resultados encontrados, que esse tipo de intervenção é bem aceito pelos alunos, pois os levam a discutir e refletir sobre suas ações.

Para finalizar destaca-se que tão importante quanto avaliar o antes e o depois da uma ação educativa realizada nesta pesquisa, é proporcionar à escola ferramentas de educação em saúde alimentar que venham a motivar os alunos na prática de hábitos alimentares saudáveis. Durante a atividade interdisciplinar com o professor de artes, os alunos deram a ideia da construção de uma lâmina (bandeja) educativa sobre a Obesidade para ser colocado no refeitório da mesma forma como é utilizado nas redes de fast-food. Tal lâmina foi elaborada pelos alunos com supervisão do professor de artes e trazia informações sobre a obesidade na forma de um jogo (Figura 1).

O presente estudo foi desenvolvido em uma escola particular, e acreditamos que a estratégia interdisciplinar possa ser utilizada em outras instituições tanto privadas como públicas de ensino, tornando a escola um espaço promotor de saúde. 


\section{Referências}

1. Freitas LK, Cunha AT Jr, Knackfuss MI, Medeiros HJ. Obesidade em adolescentes e as políticas públicas de nutrição. Cien Saude Colet. 2014;19(6):1755-62. http://dx.doi.org/10.1590/1413-81232014196.12382013. PMid:24897476.

2. Enes CC, Slater B. Obesidade na adolescência e seus principais fatores determinantes. Rev Bras Epidemiol. 2010;13(1):16371. http://dx.doi.org/10.1590/S1415-790X2010000100015. PMid:20683564.

3. Kotecha PV, Patel SV, Baxi RK, et al. Dietary pattern of schoolgoing adolescents in urban Baroda, India. J Health Popul Nutr. 2013;31(4):490-6. PMid:24592590.

4. Instituto Brasileiro de Geografia e Estatística (IBGE). Pesquisa de Orçamento Familiar (POF). Rio de Janeiro: IBGE; 2010. [citado em 2015 Out 06]. Disponível em: https://biblioteca.ibge.gov.br/visualizacao/livros/liv50063.pdf.

5. Brasil. Ministério da Saúde. Centro Brasileiro de Análise e Planejamento. Pesquisa Nacional de Demografia e Saúde da Criança e da Mulher: PNDS. Brasília: Ministério da Saúde; 2006.

6. Cunha DB, Souza BSN, Pereira RA, Sichieri R. Effectiveness of a randomized school-based intervention involving families and teachers to prevent excessive weight gain among adolescents in Brazil. PLoS One. 2013;8(2):e57498. http://dx.doi. org/10.1371/journal.pone.0057498. PMid:23451237.

7. World Health Organization Europe Report (WHOER). First Conference of European Network of Health promoting schools. Thessaloniki: WHO; 1997.

8. Brasil. Ministério da Educação. Base Nacional Comum Curricular. Proposta Preliminar. Segunda versão revista. Brasília: MEC; 2016 [citado em 2017 Set. 04]. Disponível em: http://historiadabncc.mec.gov.br/documentos/bncc-2versao.revista. pdf.

9. Lima MBMP. Percepção sobre alimentação e obesidade em adolescentes de escolas públicas do Distrito Federal [monografia]. Brasília: Universidade de Brasília; 2014. 33 p.

10. Toral N, Conti MA, Slater B. Alimentação saudável na ótica dos adolescentes: percepções e barreiras à sua implementação e características esperadas em materiais educativos. Cad Saude Publica. 2009;25(11):2386-94. http://dx.doi.org/10.1590/ S0102-311X2009001100009. PMid:19936477.

11. Santos KMO, Barros AA Fo. Fontes de informação sobre nutrição e saúde utilizadas por estudantes de uma universidade privada de São Paulo. Rev Nutr. 2002;15(2):201-10. http://dx.doi.org/10.1590/S1415-52732002000200009.

12. Nogueira BPT, Barbosa ADGB. Análise da percepção de adolescentes sobre obesidade em escolas públicas de Fortaleza. Rev. Bras. Fisiologia do Exercício. 2015;14(1):28-37.

13. Serrano SQ, Vasconcelos MG, Silva GA, Cerqueira MM, Pontes CM. Percepção do adolescente obeso sobre as repercussões da obesidade em sua saúde. Rev Esc Enferm USP. 2010;1(44):25-31. PMid:20394215.

\section{Contribuição dos autores}

Elen Lemões Mota de Almeida: Contribuiu substancialmente para a concepção e o planejamento da pesquisa, para a análise e interpretação dos dados, bem como contribuiu significativamente na elaboração do manuscrito. Renato da Costa Teixeira: Contribuiu para a concepção e o planejamento da pesquisa, com orientação metodológica, com a análise e a interpretação dos dados, e na revisão crítica do manuscrito. 